\title{
Tunable Reflectarray Cell for Wide Angle Beam-Steering Radar Applications
}

\author{
F. Venneri, S. Costanzo, and G. Di Massa \\ Dipartimento di Ingegneria Informatica, Modellistica, Elettronica e Sistemistica, Università della Calabria, 87036 Rende (CS), Italy \\ Correspondence should be addressed to S. Costanzo; costanzo@deis.unical.it
}

Received 10 May 2013; Accepted 1 July 2013

Academic Editor: Alvaro Rocha

Copyright (C) 2013 F. Venneri et al. This is an open access article distributed under the Creative Commons Attribution License, which permits unrestricted use, distribution, and reproduction in any medium, provided the original work is properly cited.

\begin{abstract}
An electronically tunable reflectarray element is proposed in this work to design beam-steering antennas useful for radar applications. A reduced size reflectarray unit cell is properly synthesized in order to extend the antenna beam scanning capabilities within a wider angular region. The radiating structure is accurately optimized to provide a full phase tuning range by adopting a single varactor load as phase shifter element. A $0.46 \lambda$-reflectarray cell is designed at the frequency of $11.5 \mathrm{GHz}$, obtaining a phase agility of about $330^{\circ}$. The cell is successfully adopted for the design of a $21 \times 21$ reconfigurable reflectarray. The antenna is numerically tested for different configurations of the varactors capacitance values, and good beam-steering performances are demonstrated within a wide angular range.
\end{abstract}

\section{Introduction}

Modern radar systems usually adopt phased array antennas as transmission/reception modules. Phased arrays integrate the actual radiating structures, consisting of an array of elementary antennas, with phase shifter components, tunable power amplifiers, and switches [1]. These additional devices allow to control the input signal of each radiating element, thus offering the capabilities to electronically steer the radiated main beam. Phased arrays offer many advantages with respect to mechanically scanned antennas, such as low profile, agile beams, and scalability. Furthermore, electronically scanned antennas offer increased data rates, instantaneous positioning of the radar beam, avoiding also mechanical vibrations, and errors associated with mechanically scanned systems.

An attractive alternative to traditional phased array antennas is offered by the reflectarray antenna concept [2]. As a matter of fact, reflectarrays may be specifically designed also for applications requiring pattern reconfigurability or beam-scanning capabilities. Reconfigurable reflectarrays may offer many advantages over conventional phased arrays, such as reduced costs and volume, a simpler architecture due to the absence of complicated beam-forming networks, and increased efficiencies due to the adoption of spatial feeding.
They consist of an array of microstrip elements illuminated by a feed antenna (Figure 1(a)). Each radiator is properly designed to compensate for the phase delay in the path coming from the feed and to introduce a phase contribution able to create a total reradiated field with some desired features, such as prescribed beam directions and/or shapes.

Many different reflectarray configurations have been proposed in the literature [2] also for $\mathrm{mm}$ waves applications [3], and recently, many efforts have been spent in the design of reconfigurable reflectarray elements, which are usually based on the use of tunable components and/or materials, such as MEMs, varactor diodes, and liquid crystal substrates [4-6].

Recently, the authors have proposed a novel tunable reflectarray element based on the use of an aperture-coupled patch electronically driven by a single varactor diode [710]. The radiating patch is coupled to a microstrip line printed onto a different substrate and loaded by a varactor (Figure 1(b)). By changing the bias voltage across the diode, the phase response of each element can be dynamically modified. A detailed description of the proposed phase control mechanism is reported in [10].

The phase tuning capabilities of the proposed reflectarray configuration have been already demonstrated in [7], while in $[8-10]$ a reflectarray prototype composed by $3 \times 15$ elements, 


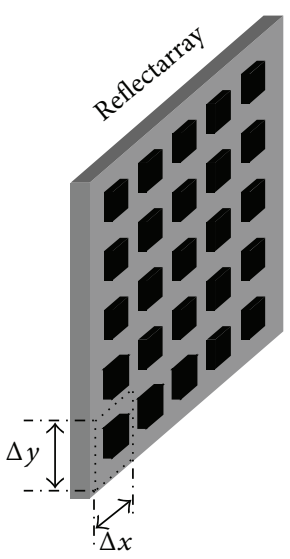

(a)
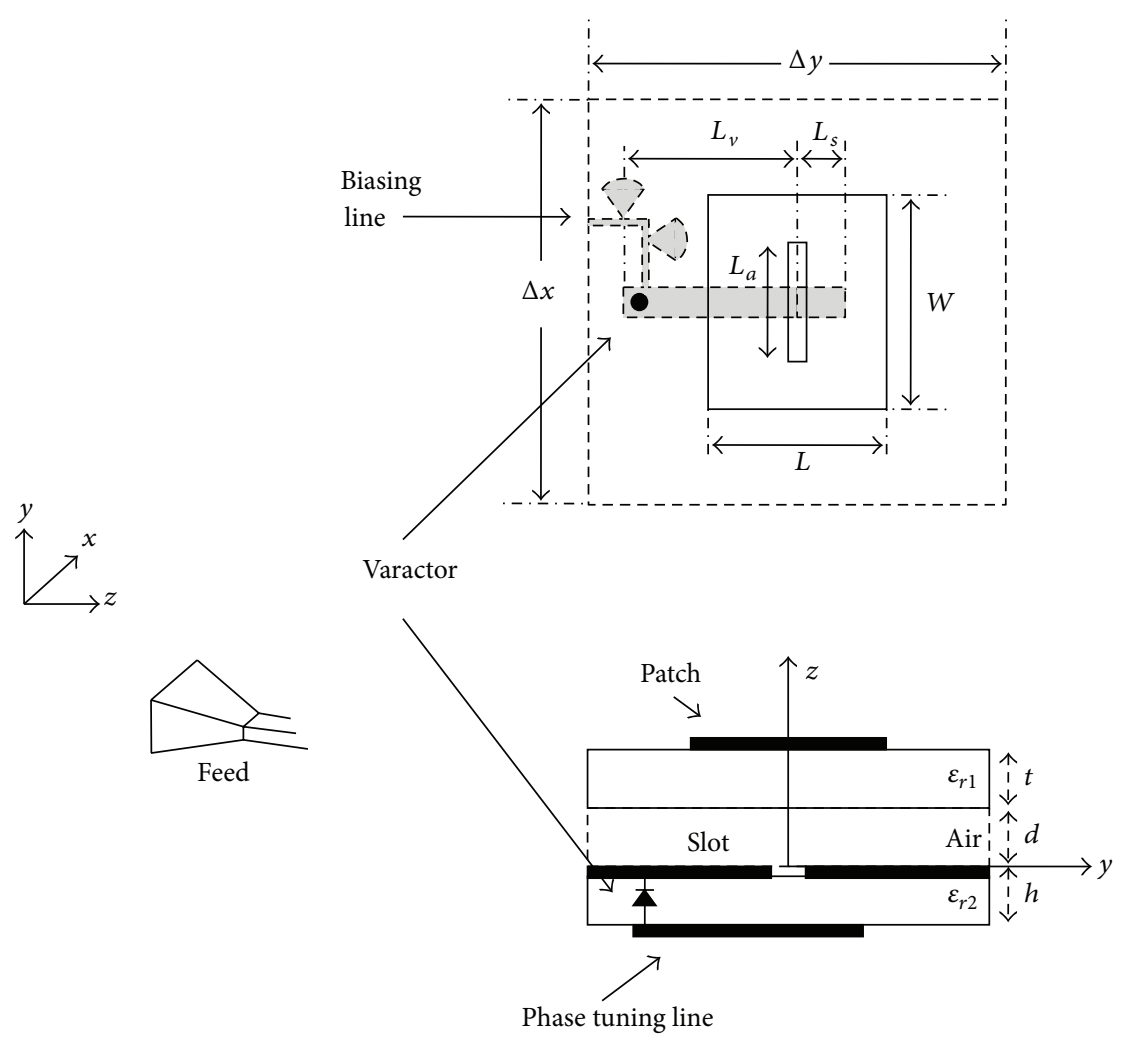

(b)

FiguRE 1: (a) Reflectarray antenna and (b) aperture-coupled reconfigurable reflectarray cell.

with a unit cell with equal to $\Delta x \times \Delta y=0.7 \lambda_{0} \times 0.7 \lambda_{0}$, has been successful designed at the frequency of $11.5 \mathrm{GHz}$. The synthesis approach described in $[11,12]$ has been adopted in order to compute the desired voltages distributions across the diodes. The antenna has been tested into the Microwave Laboratory at the University of Calabria, equipped with both near-field $[13,14]$ and far-field facilities. Various measurements of its radiation pattern for different configurations of the varactors biasing voltages have been performed, thus demonstrating in [10] the reconfiguration capabilities of the fabricated reflectarray prototype. In particular, good beam-steering performances have been obtained within an angular region going from $-25^{\circ}$ up to $25^{\circ}$. In this work, the reflectarray cell proposed in [7-10] is properly redesigned in order to enlarge the allowable beam scanning area, so to give the opportunity for designing wide-angle beam-steering antennas, suitable for radar applications. Pointing out that a large scan angle requires a close element spacing, less than or equal to half wavelength at the operating frequency [15], a reflectarray unit cell with a reduced size equal to $0.46 \lambda_{0} \times$ $0.46 \lambda_{0}\left(f_{0}=11.5 \mathrm{GHz}\right)$ is proposed. In order to accommodate the phasing circuitries inside the reduced available area embedded in the unit cell, the antenna stratification layers are properly modified by choosing a higher permittivity for the phasing line substrate. According to the considerations reported in [10], the varactor loaded line is accurately resized in order to maximize the antenna phase agility. A phase tuning range of about $330^{\circ}$ is numerically demonstrated, by varying the capacitance of the varactor diode within the values ranging from $0.2 \mathrm{pF}$ up to $2 \mathrm{pF}$.

The designed cell is adopted to synthesize a $21 \times 21$ reflectarray antenna able to steer the main beam up to $65^{\circ}$, as assessed numerically.

\section{Design of a Reconfigurable Reflectarray Element Embedded into a Unit Cell with Reduced Size}

2.1. Performance Limitations of Beam-Steering Arrays. The angular displacement of an electronically scanned radar beam is practically limited by two main factors, namely, the element pattern and the array elements spacing. As a matter of fact, the radiation pattern of an array of identical radiators is given by the product of the array factor and the element pattern. If the single array radiator is isotropic, that is, the array elements radiate an electric field quite uniform along those directions belonging to the scanning plane, only the array factor will affect the total radiation pattern.

However, practical array element patterns are not omnidirectional, showing an amplitude that decays when moving away from the broadside direction. In these cases, the single element will significantly reduce the amplitude of the scanned beam, except in the zone where it is nearly isotropic [15]. 
The second limitation, namely, the array elements spacing, is more relevant. As a matter of fact, it is well known that a large scan angle requires a close element spacing, in order to avoid grating lobes appearance. The maximum scan angle, that a linear phased array can achieve, may be derived from the well-known relation [15]:

$$
\theta_{s \max }=\sin ^{-1}\left(\frac{\lambda}{d}-1\right)
$$

where $\theta_{s \max }$ is the maximum scan angle from broadside direction, $d$ is the spacing between two adjacent elements, and $\lambda$ is the operating wavelength. Equation (1) is derived from the array factor expression of a linear array placed along the $x$-axis or $y$-axis, and its validity can be extended to the principal cuts of a planar array placed in the $x-y$ plane [15]. If the array scan angle exceeds the value imposed by (1), grating lobes will appear along other directions. This last behavior is clearly illustrated in Figure 2, which shows the scanning limitations of a $0.7 \lambda$-spaced array. In this case, if the scan angle $\theta_{s}$ is greater than $\theta_{s \max }=25^{\circ}$, as given by (1), the array factor will show a grating lobe having the same amplitude of the scanned main beam (Figure 2(c)).

Equation (1) also states that half wavelength spaced arrays will have a complete theoretical scan range of $\pm 90^{\circ}$, as illustrated in Figure 3.

The maximum scan angle achievable by a phased array is also a function of the array length and the desired half-power beam width [15]; however, the condition imposed by (1) is necessary for the design of an array with prescribed beamsteering capabilities.

2.2. Reflectarray Element Design. In order to improve the scanning capabilities of the reconfigurable reflectarray configuration proposed in [7-10], the single reflectarray element is properly redesigned by reducing the unit cell size. In fact, as discussed in the previous paragraph, a closer array elements spacing assures a larger scanning region.

The unit cell dimension is fixed to a value less than half wavelength at the operating frequency $f_{0}=11.5 \mathrm{GHz}$. In particular, the array grid size $\Delta x \times \Delta y$ is set to $0.46 \lambda_{0} \times$ $0.46 \lambda_{0}$. Furthermore, as demonstrated in [16-18], a reduced unit cell size allows to improve the bandwidth performances of reflectarray antennas. This last aspect is not considered in the present paper, but the relative analysis will be performed in a future work.

In order to allow the accommodation of the tuning circuitries in the smaller area embedded into the unit cell, the phasing line substrate adopted in [7] is properly substituted with a dielectric layer with $\varepsilon_{r}=6$ and thickness $h=0.762 \mathrm{~mm}$ (see Table 1). As a matter of fact, the use of a substrate with a higher permittivity allows to reduce the wavelength inside the printed lines, thus providing the possibility to design a shorter phase tuning line. As reported in Table 1, the other layers composing the antenna stratification are equal to those adopted in [7].

The reflectarray unit cell is synthesized through a fullwave simulation code (Ansoft Designer) based on the method of moments. The infinite array approach is adopted in order

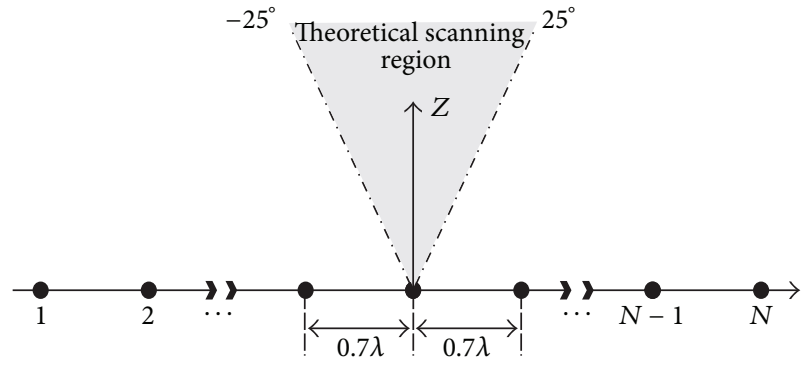

(a)

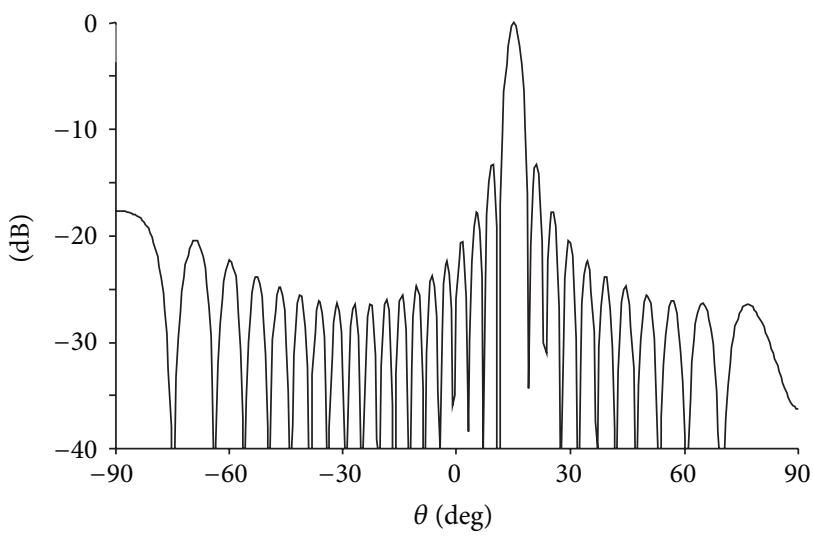

(b)

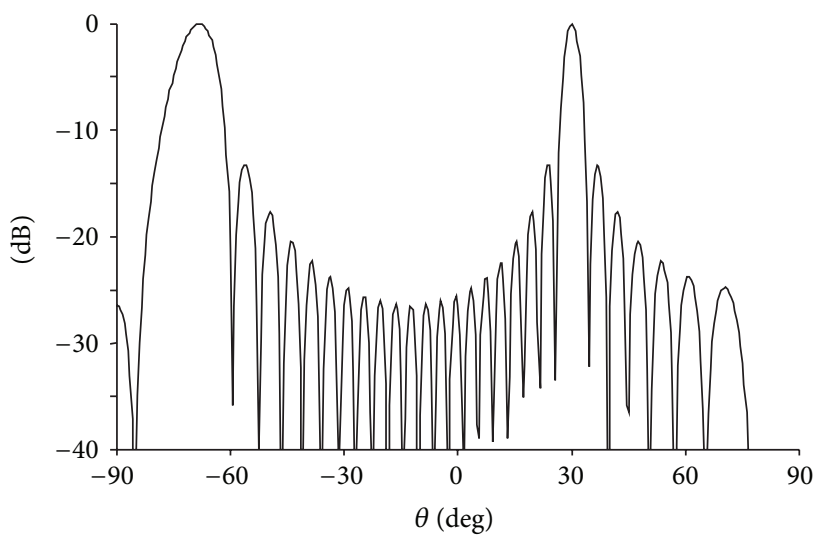

(c)

FIGURE 2: Scanning performances of an $N$-elements linear array with spacing equal to $0.7 \lambda$ : (a) allowable scanning area given by relation (1); (b) array factor for a scan angle $\theta_{s}=15^{\circ}$ and $N=21$; (c) array factor for a scan angle $\theta_{s}=30^{\circ}$ and $N=21$.

to take into account the mutual coupling effects, relevant for the assigned reduced interelement spacing.

The radiating structure design is performed by a proper tuning of patch and slot sizes, with the aim to satisfy the resonance condition as well as the matching between the patch and the phasing line, at the operating frequency of $11.5 \mathrm{GHz}$.

A varactor diode, with a tunable capacitance $C_{v}$ ranging from $0.2 \mathrm{pF}$ to $2 \mathrm{pF}$, is integrated to the microstrip line in order to obtain the required reconfiguration capabilities. The varactor diode is modeled with the equivalent circuit 


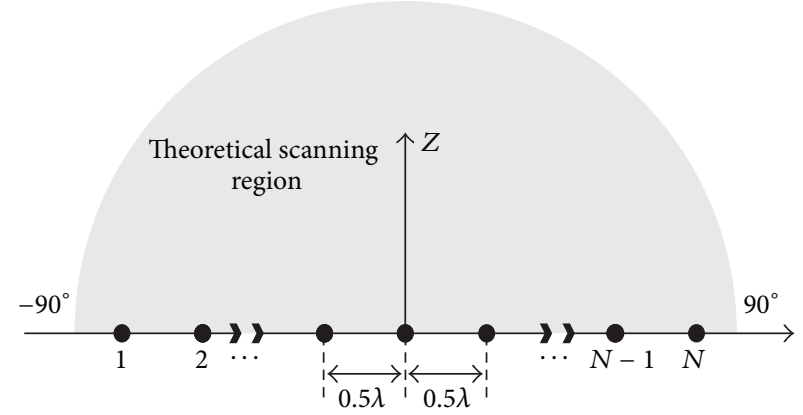

(a)

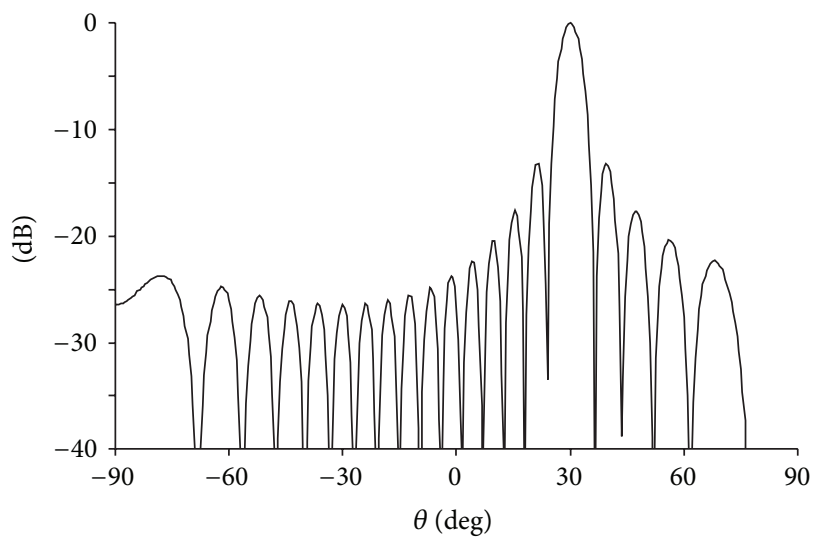

(c)

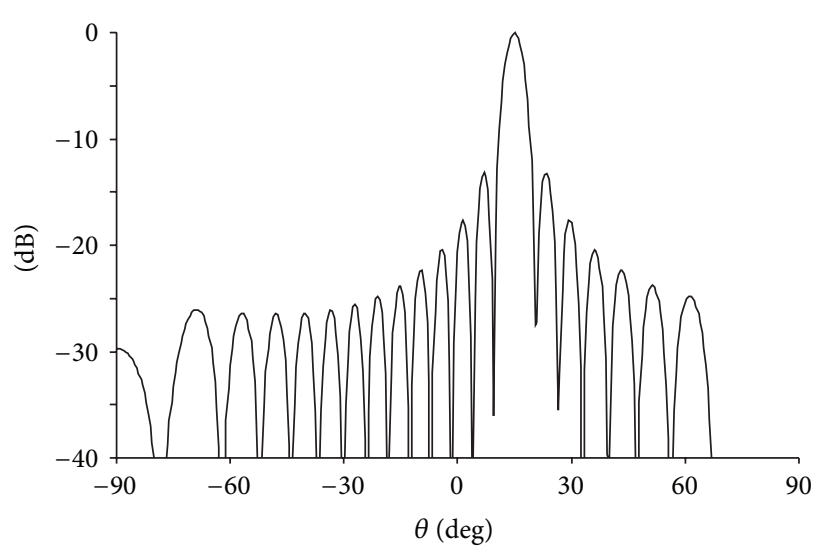

(b)

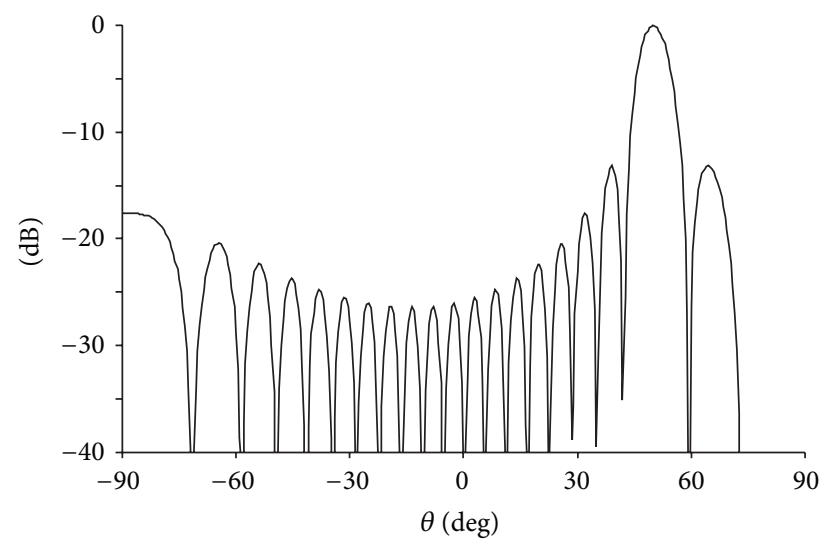

(d)

FIGURE 3: Scanning performances of an $N$-elements linear array with spacing equal to $0.5 \lambda$ : (a) allowable scanning area given by relation (1); (b) array factor for a scan angle $\theta_{s}=15^{\circ}$ and $N=21$; (c) array factor for a scan angle $\theta_{s}=30^{\circ}$ and $N=21$; (d) array factor for a scan angle $\theta_{s}=50^{\circ}$ and $N=21$.

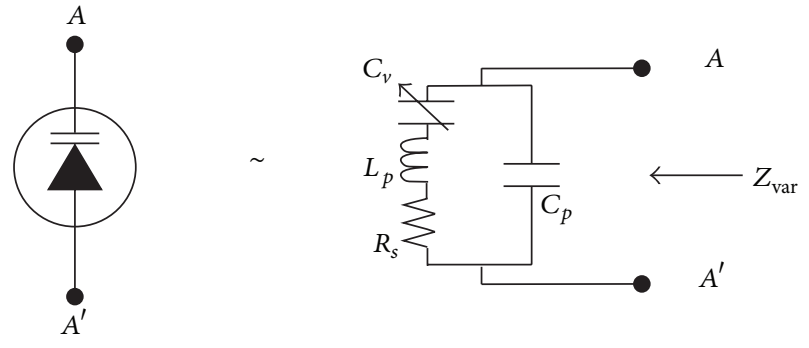

FIGURE 4: Equivalent circuit model of a varactor diode.

illustrated in Figure 4, which takes into account the package parasitic effects $\left(L_{p}, C_{p}\right)$ and the diode losses $\left(R_{s}\right)$. The varactor lumped parameters are fixed to the following values, derived by the Microsemi MV31011-89 diode datasheet: $L_{p}=$ $0.2 \mathrm{nH}, C_{p}=0.15 \mathrm{pF}$, and $R_{s}=1.36 \Omega$.

As described in [10], the two line sections $L_{v}$ and $L_{s}$ (see Figure 1(b)) are optimized in order to maximize the phase agility of the element for the assigned varactor capacitance range. At this purpose, a parametric analysis of the reflectarray element is performed with respect to the lengths $L_{v}$ and $L_{s}$, by assuming a normally incident plane wave. Figure 5 shows the reflection phase curves versus the varactor capacitance, computed for different values of the line length.

It can be observed that by increasing $L_{s}$, for a fixed value of $L_{v}$ (Figure 5(a)), a higher phase tuning range is obtained. As accurately demonstrated in [10], this last result is due to the introduction of a proper inductive effect, which is directly related to the stub length. As a proof of this concept, the input impedance $Z_{t^{\prime}}$ of the designed aperture coupled patch, evaluated at the slot center, is reported under Figure 6, for different values of the stub length $L_{s}$ ranging from $1.05 \mathrm{~mm}$ (the matched case) up to $5.2 \mathrm{~mm}$. It can be observed that for an increased input reactance a wider phase tuning range is achieved (Figure 5(a)). In particular, a phase tuning of about $330^{\circ}$ is obtained for $L_{s}=5.2 \mathrm{~mm}$. On the other hand the length $L_{v}$ is tuned in order to match the maximum phase variation with the available varactor capacitance range [10]. As a matter of fact, Figure 5(b) shows that for any fixed value of $L_{s}$, the section $L_{v}$ can be chosen to shift the phase curve within the capacitance range with the aim to increase the allowable phase tuning range, so obtaining a phasing line acting as a $360^{\circ}$ phase shifter. 


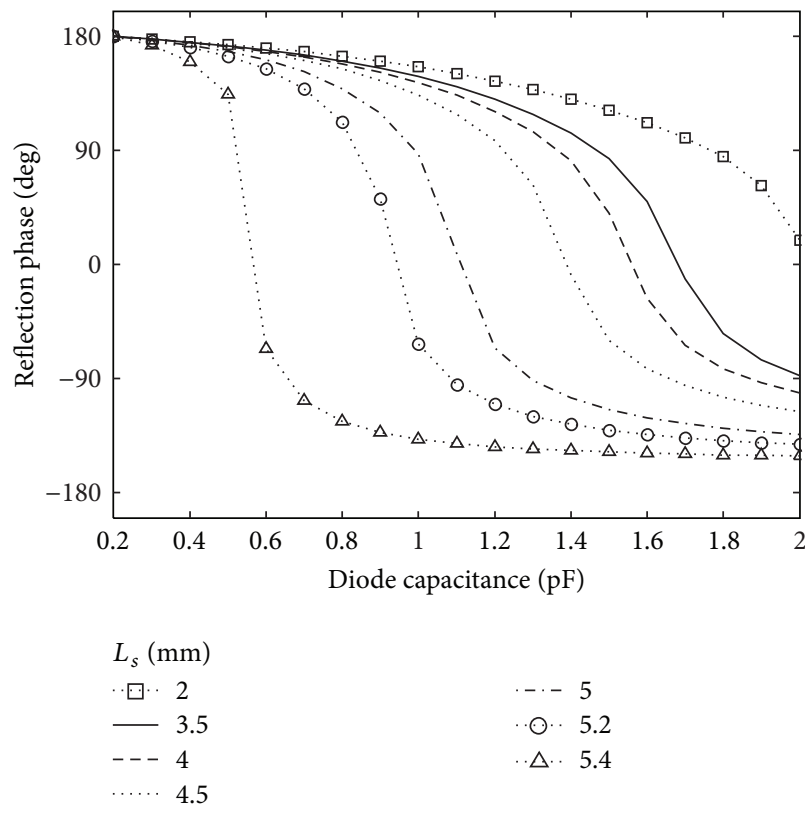

(a)

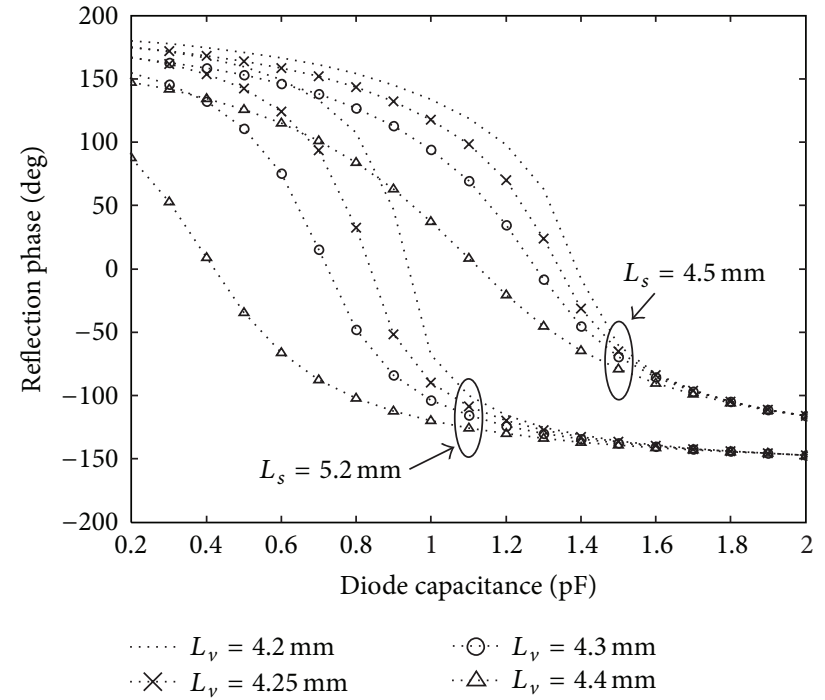

(b)

Figure 5: Phase curves versus diode capacitance for different line lengths: (a) $L_{v}=4.2 \mathrm{~mm}$ and $L_{s}$ ranging from $2 \mathrm{~mm}$ up to $5.4 \mathrm{~mm}$; (b) $L_{v}$ ranging from $4.2 \mathrm{~mm}$ up to $4.4 \mathrm{~mm}$ for some fixed values of $L_{s}$.

TABLE 1: Element stratification.

\begin{tabular}{|c|c|c|c|c|}
\hline \multirow[t]{2}{*}{ Layer } & $\begin{array}{l}\text { Element designed in [7] } \\
\Delta x \times \Delta y=0.7 \lambda_{0} \times 0.7 \lambda_{0}\end{array}$ & \multicolumn{3}{|c|}{$\begin{array}{l}\text { Element designed in this work } \\
\Delta x \times \Delta y=0.46 \lambda_{0} \times 0.46 \lambda_{0}\end{array}$} \\
\hline & Material & Thickness & Material & Thickness \\
\hline Patch & Copper & $35 \mu \mathrm{m}$ & Copper & $35 \mu \mathrm{m}$ \\
\hline \multirow{2}{*}{ Antenna substrate } & $\varepsilon_{r 1}=2.33$ & $t=0.762 \mathrm{~mm}$ & $\varepsilon_{r 1}=2.33$ & $t=0.762 \mathrm{~mm}$ \\
\hline & Air & $d=0.762 \mathrm{~mm}$ & Air & $d=0.762 \mathrm{~mm}$ \\
\hline Ground plane with slot & Copper & $35 \mu \mathrm{m}$ & Copper & $35 \mu \mathrm{m}$ \\
\hline Phasing line substrate & $\varepsilon_{r 2}=2.33$ & $h=0.762 \mathrm{~mm}$ & $\varepsilon_{r 2}=6$ & $h=0.762 \mathrm{~mm}$ \\
\hline Phasing line & Copper & $35 \mu \mathrm{m}$ & Copper & $35 \mu \mathrm{m}$ \\
\hline
\end{tabular}

The dimensions of the optimized reflectarray element are reported in Table 2 .

Furthermore, the same table shows that the designed tuning line is shorter than the line controlling the reflectarray element described in [7]. In particular, a 35\% length reduction is obtained, thus allowing the allocation of the tuning circuitries inside the smaller $0.46 \lambda_{0} \times 0.46 \lambda_{0}$ cell $(12 \mathrm{~mm} \times$ $12 \mathrm{~mm}$ at $11.5 \mathrm{GHz}$ ).

The simulated element pattern of the designed unit cell is reported in Figure 7. The depicted diagrams refer to the reflectarray element with a phasing line having dimensions $L_{v}=4.2 \mathrm{~mm}$ and $L_{s}=5.2 \mathrm{~mm}$. The radiation patterns computed in the two principal planes show a nearly isotropic behavior within the range from $-45^{\circ}$ up to $45^{\circ}$, as in the case of a typical $\cos (\theta)$ source.

In conclusion, the proposed unit cell could be suitable for the design of reflectarray antennas with improved beamsteering capabilities, as the main beam could be scanned within a quite large angular region ranging from $-45^{\circ}$ up to $45^{\circ}$, without occurring in the grating lobes phenomena. Furthermore, within this scanning range the main lobe will have at most a $3 \mathrm{~dB}$ amplitude reduction, as demonstrated by the simulated element pattern (Figure 7).

\section{Design of a Beam-Steering Reflectarray}

In order to prove the effectiveness of the proposed cell, a $21 \times 21$ reflectarray is designed at the frequency of $11.5 \mathrm{GHz}$. The array is illuminated by a broadside feed placed at a distance of $34 \mathrm{~cm}$. A synthesis algorithm [11] is adopted in order to compute the varactor capacitance values which allow to steer the radiated main beam from $0^{\circ}$ up to $65^{\circ}$ in the $H$ plane. The computed radiation patterns depicted in Figure 8 show the validity of the proposed approach, as the main beam is successfully moved along the desired directions. As expected by the array theory, Figure 8 shows that the main beam amplitude decreases when moving away from the broadside direction. In particular, it can be observed that 
TABLE 2: Element dimension.

\begin{tabular}{lcc}
\hline & Element designed in [7] & Element designed in this work \\
& $\Delta x \times \Delta y=0.7 \lambda_{0} \times 0.7 \lambda_{0}$ & $\Delta x \times \Delta y=0.46 \lambda_{0} \times 0.46 \lambda_{0}$ \\
\hline Patch size & $W \times L=8.2 \mathrm{~mm} \times 9.3 \mathrm{~mm}$ & $W \times L=7.75 \mathrm{~mm} \times 7.75 \mathrm{~mm}$ \\
Slot size & $L_{a} \times W_{a}=5.8 \mathrm{~mm} \times 0.6 \mathrm{~mm}$ & $L_{a} \times W_{a}=5.7 \mathrm{~mm} \times 0.5 \mathrm{~mm}$ \\
Phase tuning & $L_{v}=6.5 \mathrm{~mm}+L_{s}=7.8 \mathrm{~mm}$, & $L_{v}=4.2 \mathrm{~mm}+L_{s}=5.2 \mathrm{~mm}$ \\
line & $W_{s}=3.07 \mathrm{~mm}$ & $W_{s}=1.6 \mathrm{~mm}$ \\
\hline
\end{tabular}

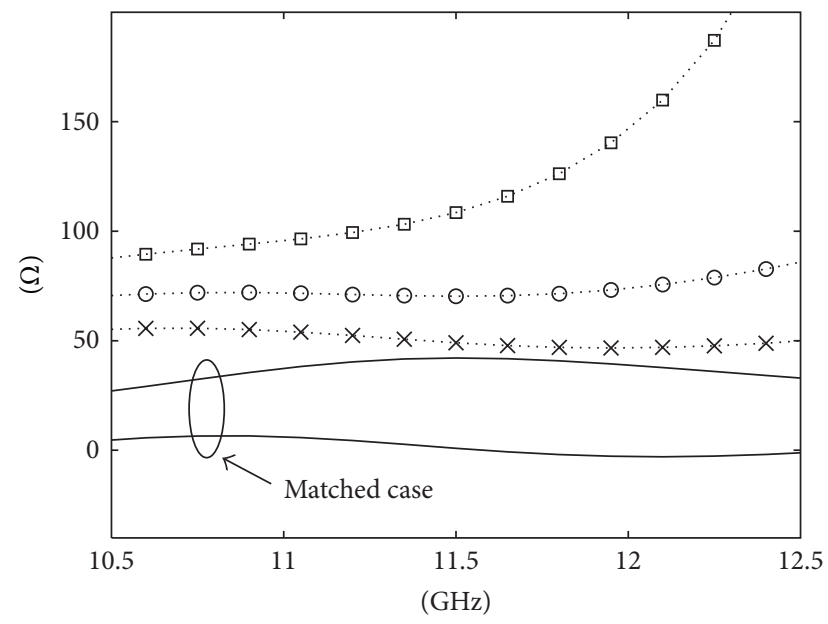

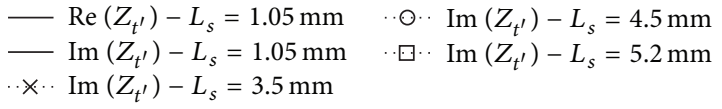

FiguRE 6: Input impedance $Z_{t^{\prime}}$ for different stub lengths.

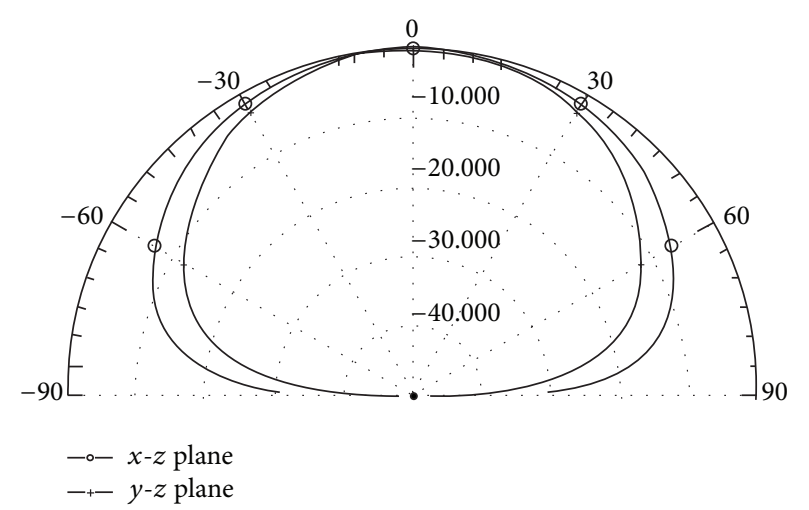

FIGURE 7: Element pattern of designed reflectarray element.

the scan loss increases away from broadside by following the cosine behavior of the element pattern. Thus, an acceptable $3 \mathrm{~dB}$ scan loss is obtained when the main lobe is pointed along the direction $\theta_{s}=45^{\circ}$, while for $\theta_{s}=65^{\circ} \mathrm{a}$ greater scan loss of about $7 \mathrm{~dB}$ is observed. Furthermore, Figure 8 shows a broader main lobe for greater scan angles. Despite limitations imposed by the array theory (i.e., scan losses and main lobe broadening), the obtained numerical results demonstrate wide angle beam-steering capabilities of the designed antenna, without occurring in grating lobes appearance.

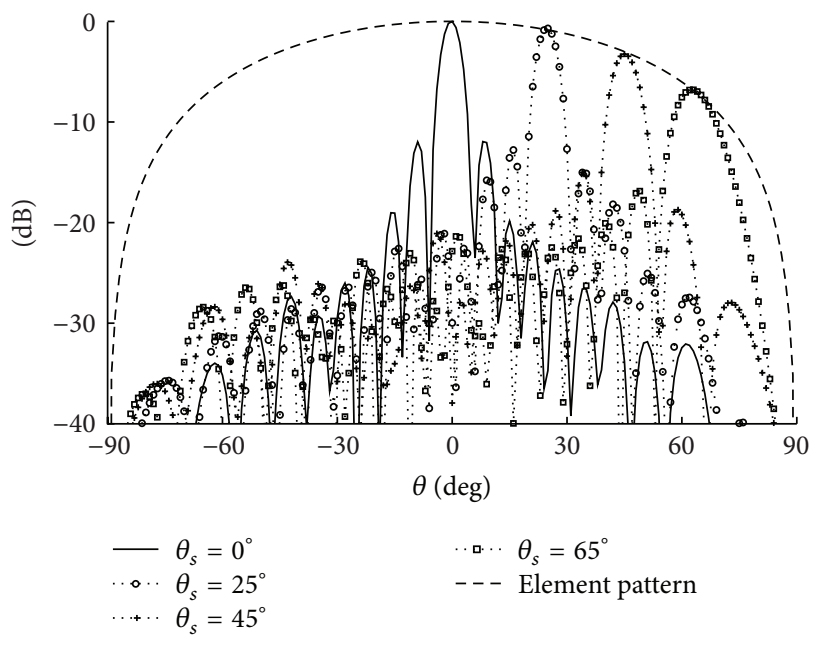

FIGURE 8: Computed radiation patterns for different configurations of the varactors capacitance values.

\section{Conclusion}

The reflectarray concept has been applied in this work to design beam-steering antennas suitable for radar applications. A reflectarray unit cell based on the use of a single varactor diode has been proposed and optimized to provide wide angle steering capabilities. At this purpose, the antenna has been properly designed by reducing the unit cell size, in order to achieve a large angular scanning. As a specific numerical example, a varactor loaded reflectarray element, embedded into a $0.46 \lambda_{0} \times 0.46 \lambda_{0}$ cell at $f_{0}=11.5 \mathrm{GHz}$, has been synthesized, obtaining a full phase tuning range of about $330^{\circ}$. The designed unit cell has been adopted to design a $21 \times 21$ reconfigurable reflectarray. The antenna has been numerically tested showing good beam-steering performances within a wide angular range from $-45^{\circ}$ up to $45^{\circ}$.

\section{Acknowledgment}

This work has been carried out under the framework of PON 01_01503 National Italian Project "Landslides Early Warning," financed by the Italian Ministry of University and Research.

\section{References}

[1] A. J. Fenn, D. H. Temme, W. P. Delaney, and W. E. Courtney, "The development of phased-array radar technology," Lincoln Laboratory Journal, vol. 12, pp. 321-340, 2000. 
[2] J. Huang and J. Encinar, Reflectarray Antennas, Wiley-IEEE Press, 2008.

[3] S. Costanzo, F. Venneri, A. Borgia, I. Venneri, and G. Di Massa, " $60 \mathrm{GHz}$ microstrip reflectarray on a benzocyclobutene dielectric substrate," IET Science, Measurement and Technology, vol. 5, no. 4, pp. 134-139, 2011.

[4] R. Sorrentino, R. V. Gatti, L. Marcaccioli, and B. Mencagli, "Electronic steerable mems antennas," in Proceedings of the 1st European Conference on Antennas and Propagation (EuCAP '06), pp. 1-8, Nice, France, November 2006.

[5] S. V. Hum, M. Okoniewski, and R. J. Davies, "Realizing an electronically tunable reflectarray using varactor diode-tuned elements," IEEE Microwave and Wireless Components Letters, vol. 15, no. 6, pp. 422-424, 2005.

[6] M. Riel and J. J. Laurin, "Design of an electronically beam scanning reflectarray using aperture-coupled elements," IEEE Transactions on Antennas and Propagation, vol. 55, no. 5, pp. 1260-1266, 2007.

[7] F. Venneri, S. Costanzo, and G. Di Massa, "Reconfigurable aperture-coupled reflectarray element tuned by single varactor diode," Electronics Letters, vol. 48, no. 2, pp. 68-69, 2012.

[8] F. Venneri, S. Costanzo, G. Di Massa, A. Borgia, P. Corsonello, and M. Salzano, "Design of a reconfigurable reflectarray based on a varactor tuned element," in Proceedings of the 6th European Conference on Antennas and Propagation (EUCAP '12), pp. 2628-2631, Prague, Czech Republic, March 2012.

[9] F. Venneri, S. Costanzo, G. Di Massa et al., "Beam-scanning reflectarray based on a single varactor-tuned element," International Journal of Antennas and Propagation, vol. 2012, Article ID 290285, 5 pages, 2012.

[10] F. Venneri, S. Costanzo, and G. Di Massa, "Design and validation of a reconfigurable single varactor-tuned reflectarray," IEEE Transactions on Antennas and Propagation, vol. 61, no. 2, pp. 635-645, 2013.

[11] F. Venneri, S. Costanzo, G. Di Massa, and G. Angiulli, "An improved synthesis algorithm for reflectarrays design," IEEE Antennas and Wireless Propagation Letters, vol. 4, no. 1, pp. 258261, 2005.

[12] S. Costanzo, F. Venneri, G. Di Massa, and G. Angiulli, "Synthesis of microstrip reflectarrays as planar scatterers for SAR interferometry," Electronics Letters, vol. 39, no. 3, pp. 266-267, 2003.

[13] S. Costanzo and G. Di Massa, "Direct far-field computation from bi-polar near-field samples," Journal of Electromagnetic Waves and Applications, vol. 20, no. 9, pp. 1137-1148, 2006.

[14] S. Costanzo and G. Di Massa, "Near-field to far-field transformation with planar spiral scanning," Progress in Electromagnetics Research, vol. 73, pp. 49-59, 2007.

[15] C. A. Balanis, Antenna Theory: Analysis and Design, John Wiley \& Sons, New York, NY, USA, 2nd edition, 1997.

[16] S. Costanzo, F. Venneri, and G. Di Massa, "Bandwidth enhancement of aperture-coupled reflectarrays," Electronics Letters, vol. 42, no. 23, pp. 1320-1321, 2006.

[17] F. Venneri, S. Costanzo, G. Di Massa, and G. Amendola, "Aperture-coupled reflectarrays with enhanced bandwidth features," Journal of Electromagnetic Waves and Applications, vol. 22, no. 11-12, pp. 1527-1537, 2008.

[18] F. Venneri, S. Costanzo, and G. Di Massa, "Bandwidth behavior of closely spaced aperture-coupled reflectarrays," International Journal of Antennas and Propagation, vol. 2012, Article ID 846017, 11 pages, 2012. 

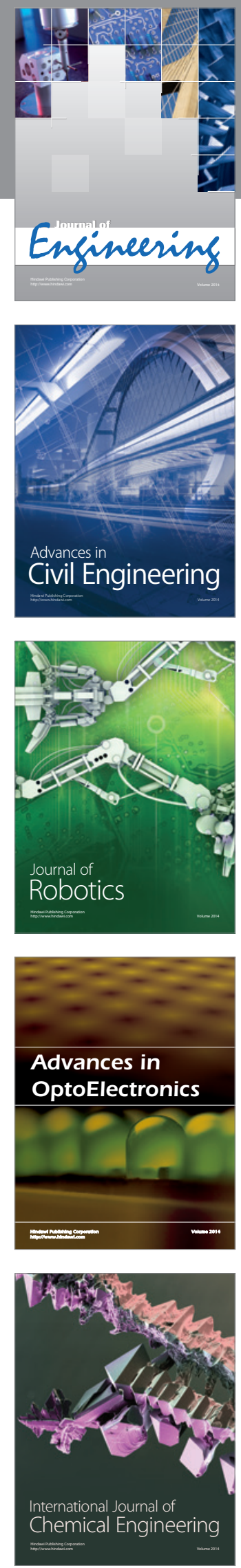

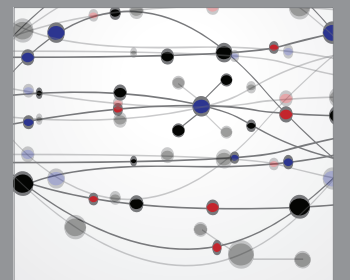

The Scientific World Journal
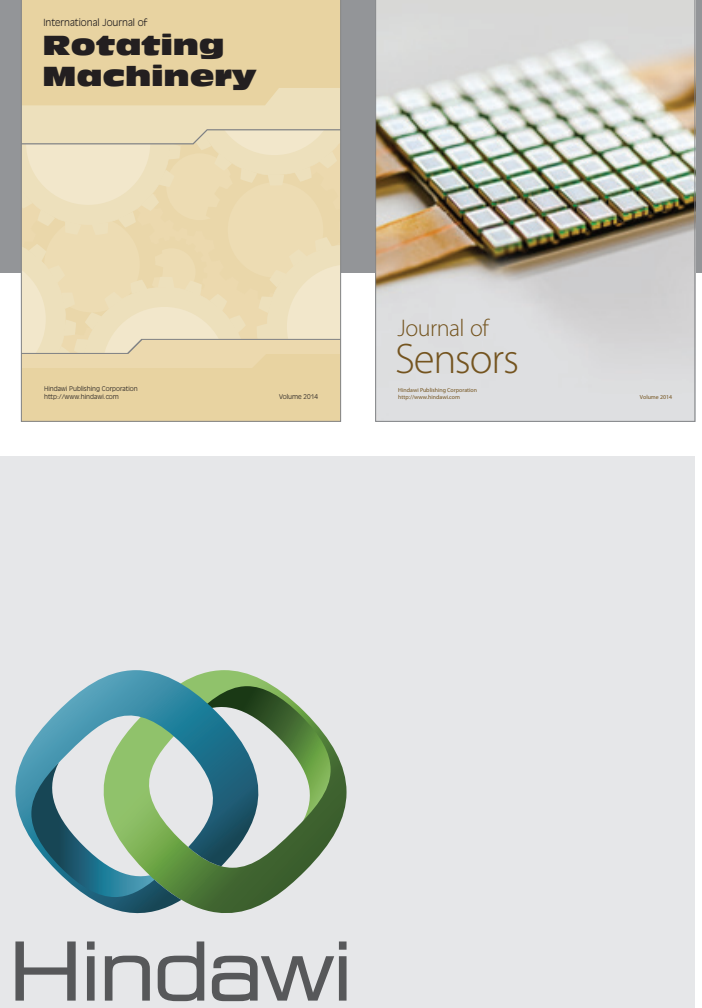

Submit your manuscripts at http://www.hindawi.com
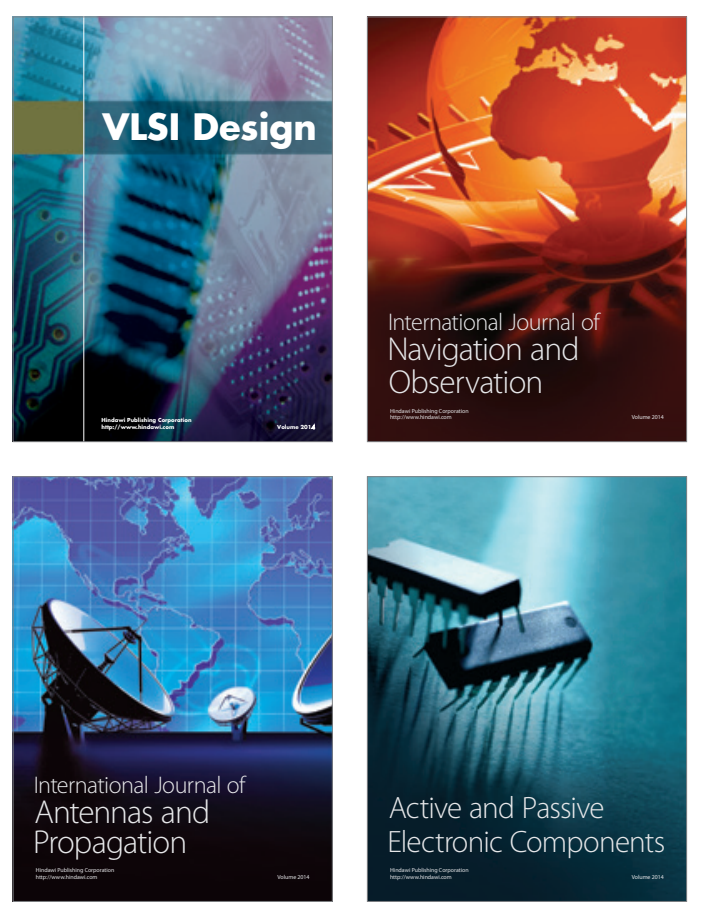
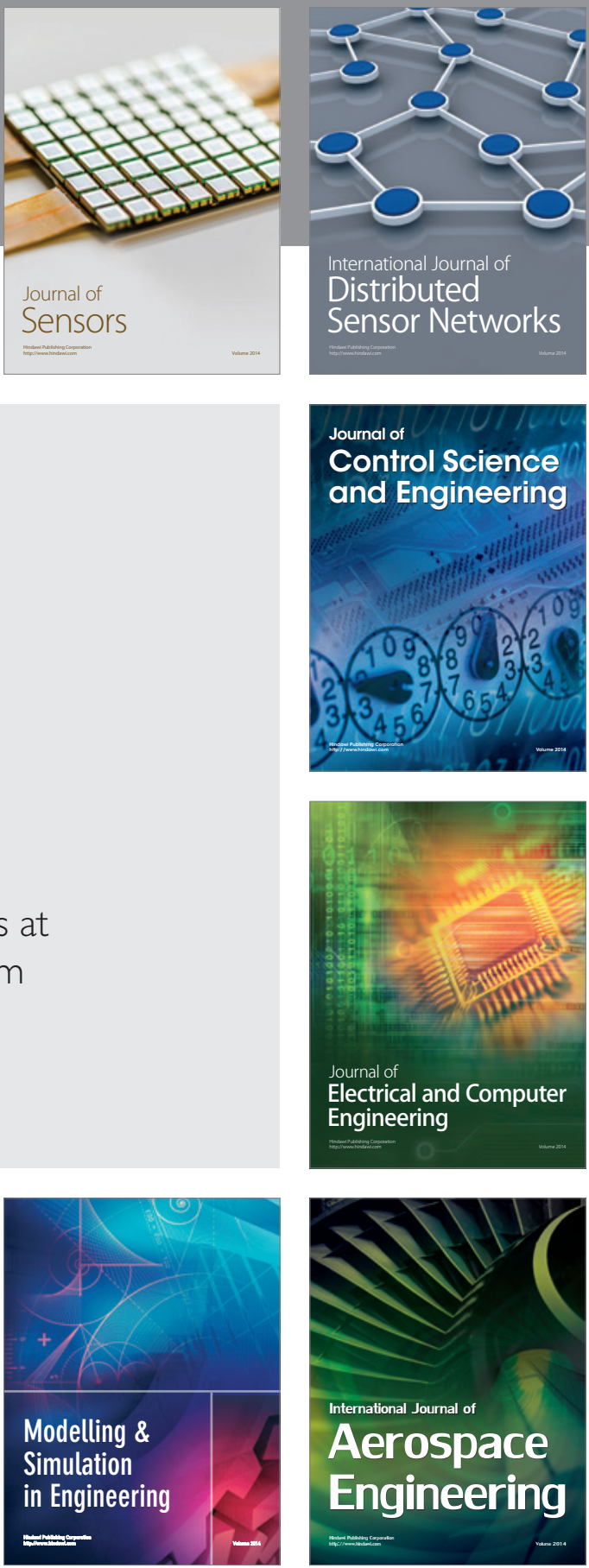

Journal of

Control Science

and Engineering
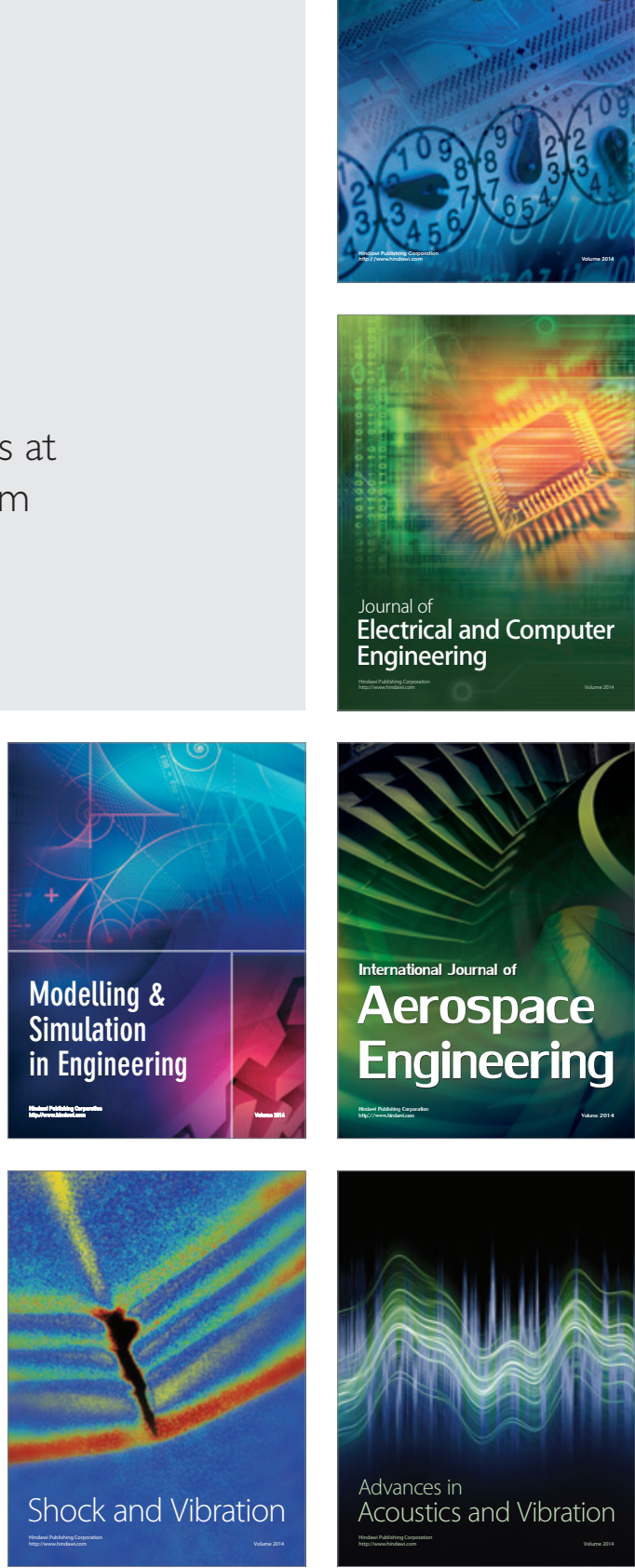